\title{
Research on Real Time Low Illumination Color Image Enhancement Method Based on FPGA
}

\author{
Huihua Jiao \\ Qiongtai Normal University, Haikou 571127, China
}

Keyword: Image enhancement; Histogram; Real time; Low illumination

\begin{abstract}
In the environment of insufficient light, video monitoring system could be easy to get indistinct videos and blurred pictures. In order to achieve better image effect, this paper proposes a method for color image enhancement in real time low illumination. By means of color space conversion, histogram equilization and linear transformation, it carries out the real time enhancement processing to the colorful video images of low illumination, broadens the dynamic range of the images, and improves the contrast and visual effect of the video images.
\end{abstract}

\section{Introduction}

Video image enhancement technology has been a very important basic processing technology in the field of image processing. The application of image enhancement technology has been applied in the fields of Aeronautics and Astronautics, military reconnaissance, medical diagnosis, satellite image processing and so on. For example, under the condition of bad weather, the image enhancement method should be used to overcome the blurred image caused by the lack of light, dust, smoke, and so on. Video monitoring system in the light of the environment is easy to lead to video blur, the image is not clear, through the image enhancement processing can improve the quality of video images, resulting in better visual effects.

Histogram equalization based on the probability theory, in order to achieve the purpose of image enhancement based on gray pixel value in the image transform, the algorithm has the typical histogram equalization algorithm (HE), histogram projection algorithm (HP), platform histogram equalization method (PE). Histogram equalization algorithm is a nonlinear stretching method, first calculate the original image histogram, and then the gray level of the cumulative probability distribution as the image transform coefficient, total gray series transformation between image grayscale conversion times. This method is mainly for the probability of a larger target pixel, pixel gray level distribution concentrated extended to wider, and the number of pixels less compression, enhance image contrast, so as to enhance the effectiveness of image.

In order to overcome the problem of lack of real-time processing existing in the prior art, the purpose of this paper is to provide a method to enhance the real-time low illumination color image, color images of low illumination for real-time enhancement, extended dynamic range image, improve the contrast at the same time, can realize the enhancement of high speed and high resolution video surveillance images, improve the visual effect of video image.

\section{Key Implementation Technology}

Implementation of Technical Solutions. The utility model relates to a real time low illumination color image enhancement method, which mainly comprises the following steps:

(1) The RGB color image is converted to $\mathrm{YCbCr}$ color space, the transformation relationship is shown as follows:

$$
\begin{aligned}
& \mathrm{Y}=0.257 * \mathrm{R}+0.564 * \mathrm{G}+0.098 * \mathrm{~B}+16 \\
& \mathrm{Cb}=-0.148 * \mathrm{R}-0.291 * \mathrm{G}+0.439 * \mathrm{~B}+128 \\
& \mathrm{Cr}=0.439 * \mathrm{R}-0.368 * \mathrm{G}-0.071 * \mathrm{~B}+128
\end{aligned}
$$

(2) $\mathrm{YCbCr}$ color space of the $\mathrm{Y}$ component histogram equalization processing; 
(3) The color components of $\mathrm{Cb}$ and $\mathrm{Cr}$ are respectively used to identify the threshold value, and the nonlinear transformation is performed for the components which do not set the threshold value;

The processed $\mathrm{YCbCr}$ color components are transformed into the RGB color space, and finally the color image is processed.

(4) Based on the above characteristics, the method of histogram equalization processing: a), histogram statistics for each pixel value of the number of statistics; b), the cumulative histogram of statistical results: histogram cumulative sum; c), the cumulative histogram histogram mapping: results of displacement and division, get the pixel histogram after mapping the value. At the same time, the threshold of $\mathrm{Cb}$ and $\mathrm{Cr}$ is determined by the normal image in advance. In addition, the conversion coefficient of the nonlinear transformation is stored in the memory in the form of a look-up table.

Implementation Scheme Design. We present a method for real-time enhancement of low illumination color images, as shown in fig. 1. All the steps in the diagram are implemented in FPGA, which includes RGB to YCbCr module, histogram equalization module, nonlinear transform module and YCbCr RGB module. The RGB YCbCr module is used to convert the RGB color image to the $\mathrm{YCbCr}$ color space. The histogram equalization module is used for histogram equalization of $\mathrm{Y}$ components in $\mathrm{YCbCr}$ color space. The nonlinear transformation module is used for judging the $\mathrm{Cb}$ and the $\mathrm{Cr}$ color components respectively, and is used to carry out the nonlinear transformation of the components which are not set in the threshold range. The $\mathrm{YCbCr} \mathrm{RGB}$ module is used to transform the processed YCbCr color components into the RGB color space.

The histogram equalization algorithm time complexity is relatively high, in order to satisfy the high-speed real-time video processing (60 frames per second) requirements, this article analyses the traditional histogram equalization method, designs a fast histogram equalization method based on FPGA, the diagram shown in fig. 2. According to the processing block diagram, the histogram equalization processing includes 3 sub modules: (1) histogram statistics module (2). Histogram accumulation module (3). The following describes the specific implementation of the 3 modules.

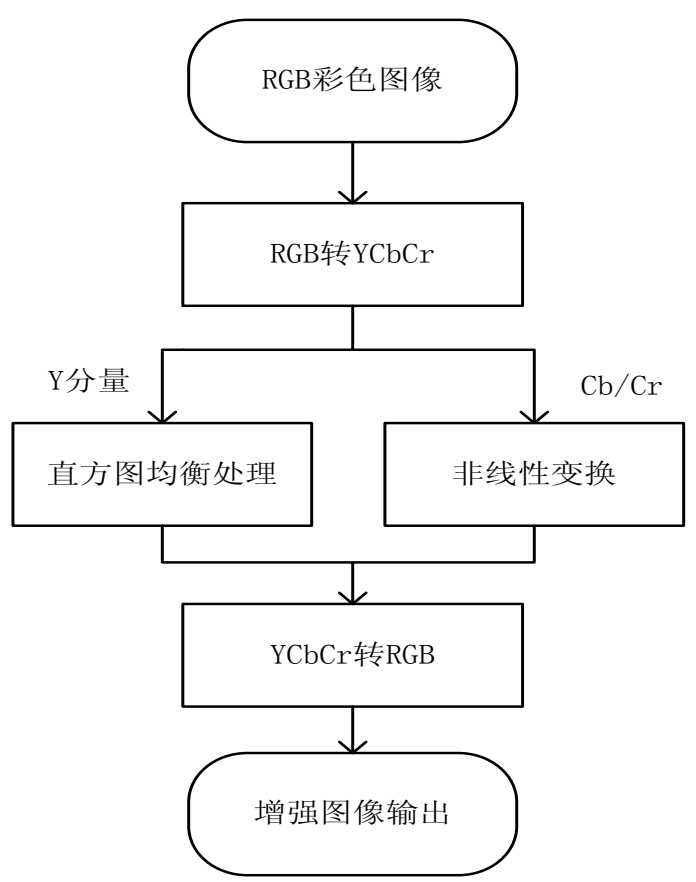

Figure 1. Real time enhancement processing flow of low illumination color image 


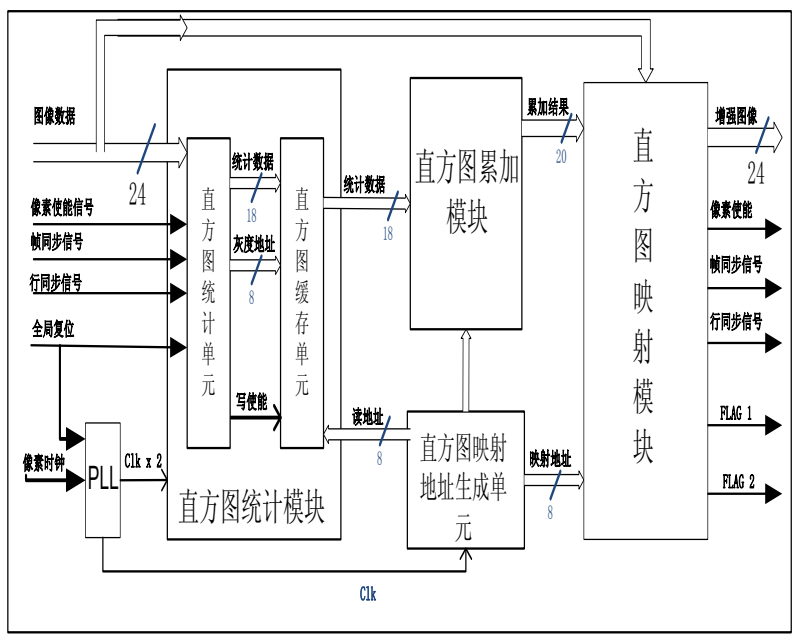

Figure 2. Block diagram of histogram equalization algorithm based on FPGA

Histogram statistics required for each pixel value of the number of statistics, so you can design a $256 \mathrm{X}$ bit 18 dual port RAM as a histogram storage unit. The input pixel value as the write address of the dual port RAM, the number of each of the same pixel plus 1, so before every write RAM first RAM in the temporary data readout, and plus 1 , and then write to RAM. Its logical structure is shown in fig. 3. Another double port RAM2 is used to preserve the final statistics of the register.

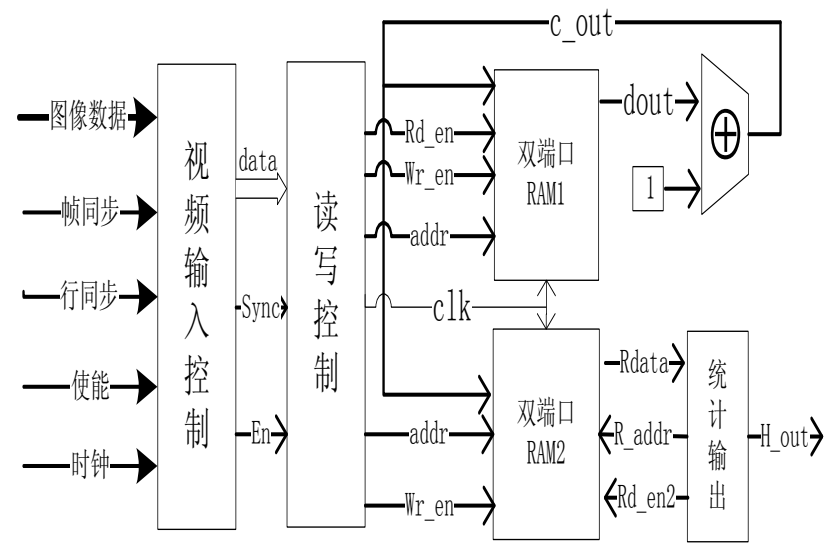

Figure 3. Logical block diagram of histogram equalization

After the end of the histogram statistics, the need for histogram statistics. And the cumulative results as a basis for equalization, so it is necessary to save and save each other in a dual port RAM. The cumulative process is from the above histogram statistical module of the dual port RAM2, in order to read out each data, and accumulated, the cumulative results are stored in the dual port RAM3. The cumulative operation is in the presence of blanking period, so after the end of each accumulation to be used to reset the sync signal. The cumulative operation is realized by accumulator in FPGA. The block diagram of the module is shown in fig. 4. 


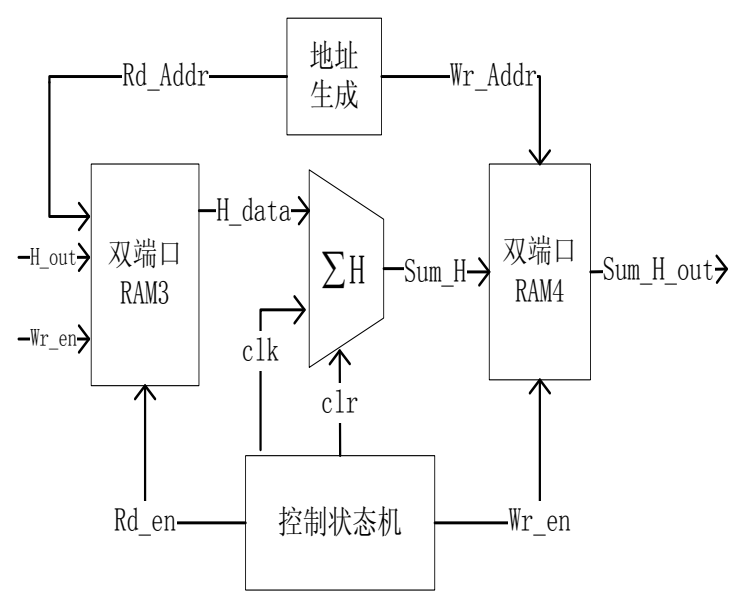

Figure 4. Histogram statistical results of cumulative processing logic block diagram

Histogram equalization algorithm can be seen before the histogram of the need for the cumulative histogram of the results of multiplication and division operations. Video source format in the scheme for $1024 X 768$ resolution, 60 per second frame rate, pixel level digital level 256. To the cumulative results of the histogram multiplied by 256 , and then $1024 X 768$. After simplification, the result can be divided into 3 parts, which is divided by a low level of 10 , and the result is the pixel value of the histogram. According to the above analysis, the realization of the histogram mapping module is shown in fig. 5.

As mentioned above, the $\mathrm{Y}$ component of $\mathrm{YCbCr}$ image histogram equalization, the threshold and nonlinear transform $\mathrm{Cr}, \mathrm{Cb}$ component, nonlinear transform coefficients can be used to advance the lookup table is stored in RAM, to achieve real-time transform. After the balanced Y component and $\mathrm{Cb}$ and $\mathrm{Cr}$ components are transformed by RGB, the enhanced color image is finally obtained.

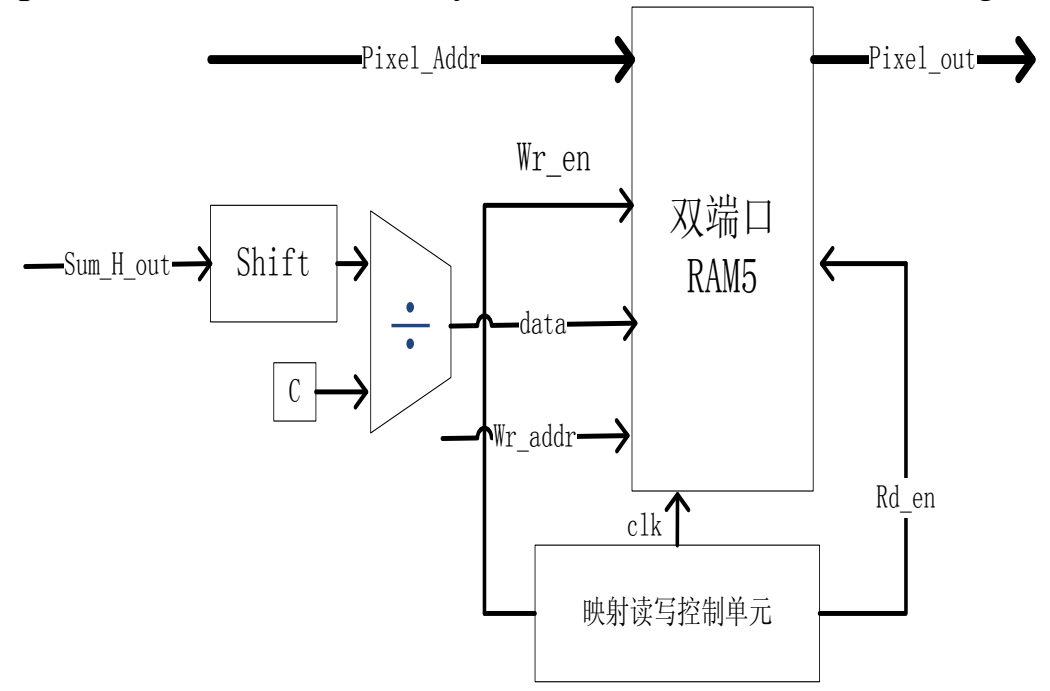

Figure 5. Histogram mapping module

Implement Device. Based on the above we design developed a real-time low illumination image enhancement device, using FPGA chip, including RGB module, YCbCr module, nonlinear transform and histogram equalization module and YCbCr RGB module; the main functions of each module are described below:

GB YCbCr module is used to convert the RGB color image to the YCbCr color space;

Histogram equalization module for the $\mathrm{YCbCr}$ color space of the $\mathrm{Y}$ component histogram equalization;

The nonlinear transform module is used to distinguish the color components of $\mathrm{Cb}$ and $\mathrm{Cr}$ respectively, which is used to transform the nonlinear components without setting the threshold value; 
The YCbCr RGB module is used to transform the processed $\mathrm{YCbCr}$ color components into the RGB color space.

On the basis of the histogram equalization module including histogram statistics module, histogram accumulation module, histogram mapping module, storage unit of the histogram module by using dual port RAM in FPGA as the statistical results of the histogram of pixel values as input address, pixel clock as a write clock, dual port RAM output port is connected with the 1 counter implementation of histogram statistics;

The cumulative histogram module uses the FPGA accumulator of statistics histogram cumulative sum, and accumulated as a result of equalization based on input and output ports of the accumulator with a dual port RAM cache, accumulated after use synchronous signal reset;

The histogram mapping module uses FPGA shift register and divider histogram mapping operations, and histogram mapping results stored in another dual port RAM, and will need to balance the values of image pixel readout address as the dual port RAM, read data into pixel values after equalization.

\section{Implementation Case Series}

The specific implementation of this scheme is implemented using Altera Arrial-II series FPGA, these modules using VHDL language, functional simulation and timing simulation by ModelSim, and finally downloaded to the FPGA online verification.

This scheme is used for the specific resolution of the low illumination color video image $1024 \times 768$ as the video source, the frame rate of 60 frame per second, the original image is shown in figure 6A. After the method of this paper to enhance the effect of treatment as shown in figure 6B. This method is suitable for real-time enhancement of low illumination color video image, and can meet the requirements of small delay, high frame rate.

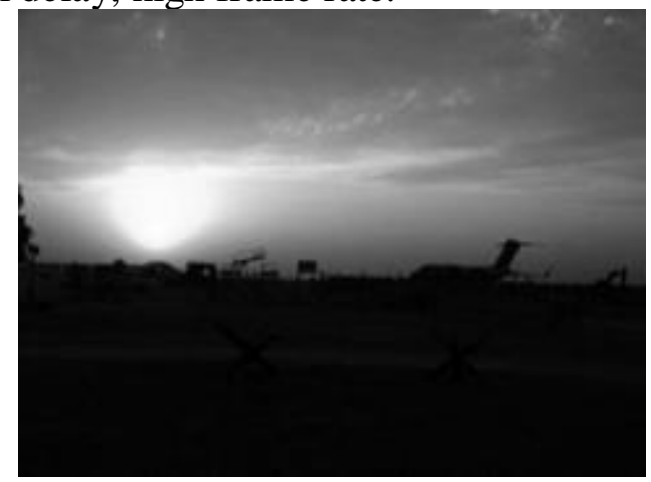

A Original image

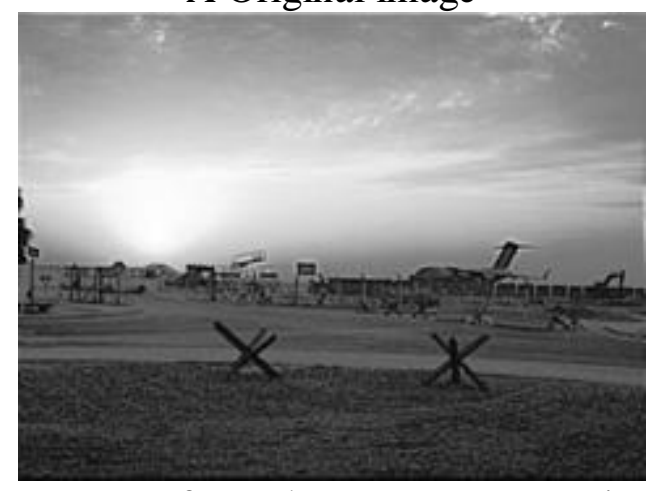

B Image after enhancement processing

Figure 6. Implementation of the case

\section{Conclusion}

We propose image enhancement method for color image enhancement of low illumination effect, high real-time processing, and image processing delay from input to output only a microsecond 
level, is very suitable for high speed real-time processing applications. The solution to the low illumination color video image real-time image enhancement, extended dynamic range, improve the contrast enhancement at the same time to realize high speed and high resolution video surveillance images, improve the visual effect of video image.

\section{Reference}

[1] Liu Zhichun, Wang Jingru. Image Enhancement Based on FPGA Design for the Pre Processor $[\mathrm{J}]$.

[2] Feng Haiqi. Hardware Implementation of Real Time Image Enhancement Processing [J]. Radio Communication Technology.1998 (3): 9-11

[3] Li Wenyong. Real Time Infrared Image Enhancement Technology [D]. Harbin Engineering University, 50-80 2012.6.

[4] Guo Qian. Improved Algorithm and Hardware Implementation of Real-time Infrared Image Enhancement Based on FPGA [D]. Nanjing University of Science and Technology, 2006.6.10-20

[5] Guo Wanchun. Realization of Real-time Digital Image Processing System Based on FPGA [D]. Harbin Engineering University, 2011.1.55-70

[6] Guo Wanchun. Research on Method of Low Illumination Adaptive Image Enhancement [J]. Information Technology 2014.10.76-81 